\title{
Evaluación de indicadores de degradación y biomasa radical en mallines de una cuenca hídrica del sur de Santa Cruz
}

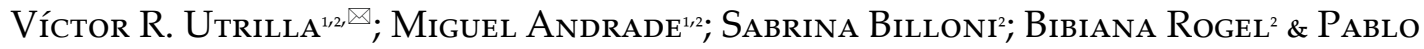 \\ L. PERI ${ }^{1 / 2}$
}

${ }^{1}$ Instituto Nacional de Tecnología Agropecuaria - EEA Santa Cruz. ${ }^{2}$ Universidad Nacional de la Patagonia Austral (UNPA).

\begin{abstract}
Resumen. Se planteó un estudio con el objetivo de analizar la asociación entre indicadores de deterioro del suelo y la vegetación, evaluar los cambios de esas variables entre sectores y condiciones de degradación, y monitorear parámetros del agua freática y la biomasa radical entre mallines con diferente condición de una cuenca hídrica del sur de Santa Cruz. En un sector húmedo bajo condición buena y en un sector húmedo y seco bajo condición moderada y muy degradada del mallín se determinaron, en dos fechas y durante tres años, la cobertura de especies con valor forrajero principal, especies indicadoras de degradación (EI), mantillo, suelo desnudo (SD), material muerto en pie y biomasa aérea disponible (B). En el suelo se determinó la resistencia mecánica (RM), la densidad aparente (Dap), el pH, la resistencia eléctrica (RE) y la materia orgánica (MO); en el agua freática, la conductividad eléctrica y la relación de absorción de sodio (RAS). En el sector húmedo del mallín se determinó la biomasa radical. Los valores de vegetación y suelo se analizaron mediante una prueba tri-factorial $2 \times 2 \times 3$ (i.e., Sector [S, Húmedo y Seco], Fecha [diciembre y abril] y Condición [C, Buena, Moderadamente Degradada y Muy Degradada]). La prueba se incluyó en un diseño completamente aleatorizado (DCA), con tres repeticiones y un arreglo en parcelas subdivididas. El estudio identificó variables de la vegetación y el suelo asociadas con la C y S del mallín. En los dos sectores bajo condición Muy Degradada se reportó mayor cobertura de EI $(P<0.05)$ y menor B $(P<0.05)$ del pastizal respecto de las condiciones restantes. La Dap fue superior $(P<0.05)$ y la biomasa radical se redujo con una mayor degradación del mallín. Se concluye que es factible analizar la asociación de variables de la vegetación y suelo con la C y S del mallín, y explicar la variación de la biomasa radical según la condición de degradación.
\end{abstract}

[Palabras clave: humedales, condición de deterioro, vegetación, suelo]

Aвstract. Indicators of degradation and root biomass for meadow ecosystems in a watershed of Southern Santa Cruz province. We conducted a study in order to analyze the association between vegetation and soil indicators, to assess the variation of these variables between sectors and condition of degradation, and to evaluate parameters of groundwater and root biomass between meadows with different condition in a watershed of southern Santa Cruz. Cover of the main forage species, litter, bare soil (BS), species that indicate degradation (IS), standing dead plant material and aerial biomass (B) were determined during three years in five contrasting meadow conditions (wet sector in good condition and wet and dry sectors in moderate and highly degraded conditions). Soil mechanical resistance, bulk density (BD), pH, electrical resistance, soil organic matter, groundwater electrical conductivity, sodium absorption ratio and root biomass were measured.

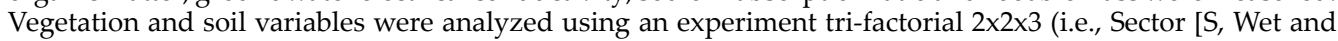
Dry], Date [December and April] and Condition [C, Good, Moderate and Highly Degraded]). A completely randomized design (CRD) with three replications and subdivided plots arrangement was used. The study identified vegetation and soil variables associated with the $C$ and S. Highly degraded meadows showed high IS and low $\mathrm{AB}$ values. $\mathrm{BD}$ was higher $(P<0.05)$ in the highly deteriorated meadow. Root biomass was reduced with the deterioration of meadow. We conclude that it is possible to analyze the associate vegetation and soil variables with the $\mathrm{C}$ and $\mathrm{S}$ of meadow and explain the variation of radical biomass in relation to condition of degradation.

[Keywords: wetlands, condition of deterioration, vegetation, soil]

Editora asociada: Patricia Kandus 


\section{INTRODUCCIÓN}

En la Patagonia, los mallines son ambientes húmedos que ocupan ca. 3-5\% de la superficie de la región y concentran entre 20 y $50 \%$ de la producción forrajera de los pastizales de esa región (Buono 2007). Los mallines de origen glacifluvial se ubican en áreas deprimidas del paisaje (Mazzoni and Vásquez 2004), lo cual favorece la acumulación hídrica en el suelo y una elevada productividad y calidad del forraje disponible para los herbívoros silvestres y domésticos (Utrilla 2003; Utrilla et al. 2005). Los principales disturbios que afectan estos ambientes se asocian con variaciones inducidas en la napa freática y el pastoreo intensivo y continuo del ganado (Raffaele 1999). En este contexto, los mallines de valle asociados a cursos de régimen nival o nivopluvial se caracterizan por una recarga del perfil edáfico que ocurre, en general, en el período otoño-primavera, momento de balances hídricos positivos, mayores caudales en cursos naturales y mayor precipitación efectiva y fusión de nieves estacionales, y también por un balance hídrico estival deficitario (Cremona et al. 1996; Peri and Monelos 1997; Horne 2010; Díaz, comunicación personal). El sobrepastoreo de los mallines disminuye la cobertura vegetal y aumenta el suelo desnudo (Bonvisutto et al. 2008; Suárez et al. 2010), reduce la producción forrajera del pastizal y el contenido del carbono orgánico edáfico total (Enriquez et al. 2015) y particulado (Enriquez and Cremona 2018), y favorece la salinización y la evaporación del agua (Bonvisutto et al. 1992; Del Valle 1993). Además, en condiciones húmedas, la presión de pastoreo elevada genera una compactación del suelo por pisoteo animal; esto resulta en aumentos en la densidad volumétrica (Enriquez et al. 2015) y en procesos de erosión hídrica de distinto tipo e intensidad (Del Valle 1993; Bonvisuto et al. 2008).

En virtud de estos disturbios, en Patagonia se plantearon varios estudios para establecer indicadores que permitan caracterizar diferentes estados de degradación de los mallines. En Río Negro y Neuquén, Bonvissuto et al. (2008) describieron cambios en la vegetación y en el suelo a través de guías de condición utilitaria para mallines andinos y extra-andinos bajo buena, regular y pobre condición. Asimismo, en mallines húmedos y secos de Río Negro, Enriquez y Cremona (2018) encontraron que el contenido de carbono orgánico edáfico particulado es un indicador ecológico adecuado para describir la degradación del suelo. En Chubut, Nakamatsu et al. (comunicación personal) identificaron la presencia de especies indicadoras de deterioro para caracterizar los mallines en distintos niveles de degradación: sin deterioro, moderadamente degradados y muy degradados. En mallines húmedos $\mathrm{y}$ secos de varias áreas ecológicas de Santa Cruz, Ormaechea et al. (2010) establecieron claves dicotómicas para identificar la condición de estos ambientes en buena, moderada y severamente deteriorados, a partir de relevar la cobertura de suelo desnudo, las especies indicadoras de deterioro y el mantillo reportados por Suárez et al. (2010). A su vez, en mallines glaciares y fluviales del sur de Santa Cruz, Vargas (2017) estableció un índice de degradación con escala entre 0 y 5 y seis estadios desde muy bajo a extremo según la cobertura y riqueza de especies indicadoras, entre otras variables florísticas. En Tierra del Fuego, Collantes et al. (2013) indicaron cambios en la composición florística de mallines húmedos y semihúmedos sometidos a largos períodos de pastoreo ovino intensivo.

Un aspecto poco estudiado en los mallines se vincula con el sistema radical de la vegetación cuya biomasa se encuentra afectada por el sobrepastoreo en mallines del norte de la Patagonia (Enriquez et al. 2015) y alterada por la compactación del suelo en virtud del pisoteo animal en humedales similares de Norte-América (Roberson 1996) y en otros ambientes de la Patagonia (Rodríguez et al. 2006). En este contexto, se planteó un estudio con el objetivo de analizar la asociación entre indicadores de deterioro de la vegetación y del suelo, evaluar los cambios de estas variables entre sectores y condiciones de degradación, y monitorear parámetros del agua freática y la biomasa radical entre mallines con diferente condición de una cuenca hídrica en la Estepa Magallánica Seca de Santa Cruz.

\section{Materiales y Métodos}

\section{Identificación del área y los sitios de estudio}

El área de estudio se ubicó en el piso de valle de la cuenca alta y media del Río Coyle, en la Estepa Magallánica Seca del Sur de Santa Cruz. Se establecieron dos sectores: Húmedo (cercano al cauce principal o secundario del mallín, con una vegetación predominante de graminoides [Carex sp., Juncus balticus y Eleocharis sp.] acompañada por gramíneas 
[Poa pratensis, Agrostis sp. y Hordeum sp.] y dicotiledóneas herbáceas [Taraxacum officinale]), y Seco (en la periferia del mallín, cubierto principalmente por Festuca pallescens acompañada por Hordeum sp., P. pratensis, J. balticus y Taraxacum officinale [Mazzoni and Vásquez 2004; Utrilla et al. 2005]). A su vez, en cada sector se identificaron tres condiciones del mallín: Buena, Moderada y Muy Degradada, según el monitoreo de las coberturas de suelo desnudo, especies indicadoras de deterioro y mantillo reportados por Suárez et al. (2010). Cabe mencionar que no fue posible identificar el sector seco y condición buena a partir de antecedentes previos en otros mallines secos afectados por el uso intensivo histórico, que genera un progresivo deterioro de la vegetación (Bonvissuto et al. 1992) y del suelo, de muy difícil recuperación (Del Valle 1993).

De esta manera, a partir de la combinación de los factores sector y condición se establecieron cinco sitios de estudio (tratamientos); cuenca alta (mallín La Tapera, LT): Húmedo-Buena,

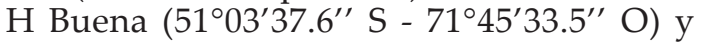
cuenca media (mallines Las Horquetas, LH): Húmedo-Moderadamente Degradado, H Mod Deg, (5123'37.4'" S - 70¹2'14.1' ' O); HúmedoMuy Degradado, H Muy Deg, (5122'49.8” S - 70¹3'38.6"'O); Seco-Moderadamente Degradado, S Mod Deg, (51²5’35.9”' S $70^{\circ} 14^{\prime} 58.9^{\prime \prime}$ O) y Seco-Muy Degradado, S Muy Deg (5122'45.7' S - 70¹3'51.1' O).

En cada sitio se evaluaron parámetros de la vegetación y físico-químicos del suelo en dos fechas (diciembre y abril), coincidentes con el inicio y final del aprovechamiento bajo pastoreo del mallín y durante tres años (2010-2011, 2011-2012 y 2012-2013). Además, en el sector húmedo y para cada condición del mallín se determinaron variables de calidad del agua freática en diciembre del primer año y abril del segundo y tercer año. La biomasa radical fue determinada en diciembre y abril del tercer año.

\section{Descripción de variables climáticas}

En los mallines LT y LH se registró la temperatura ambiental diaria con un datalogger (Modelo: HOBO TEMPERATURE, RH (C1996 ONSET), con una hora de intervalo. Las lluvias se registraron con pluviómetro de campo (fuente: datos propios y de Díaz et al. 2016). En este contexto, las temperaturas medias mensuales en los mallines descriptos oscilaron desde $9.5^{\circ} \mathrm{C}$ y $15.5^{\circ} \mathrm{C}$ (diciembre) hasta $9.0^{\circ} \mathrm{C}$ y $13.0^{\circ} \mathrm{C}$ (marzo), con valores máximos que variaron desde $11.0{ }^{\circ} \mathrm{C}$ hasta $16.0{ }^{\circ} \mathrm{C}$ en enero, y una diferencia de 2.0 ${ }^{\circ} \mathrm{C}$ a favor de los mallines $\mathrm{LH}$ respecto al mallín LT. La pluviometría anual (enero a diciembre) registrada en los años 2011 y 2012 fue, respectivamente, de 139.5 y $266.0 \mathrm{~mm}$ (LT) y de 187.0 y $272.0 \mathrm{~mm}(\mathrm{LH})$, determinada a partir de registros propios durante el estudio y otros procedentes del Banco de Datos Hidrometeorológicos de Santa Cruz (Díaz et al. 2016). Asimismo, en LT, la distribución pluvial en las cuatro estaciones fue homogénea en el 2011 (rango entre 30.0 y $40.0 \mathrm{~mm}$ ) y otoñal en el 2012 (83.5 mm). En cambio, en LH las lluvias se concentraron durante el invierno del 2011 (96.0 $\mathrm{mm}$ ) y predominaron en el verano y otoño del 2012 (88.0 y $112.0 \mathrm{~mm}$, respectivamente).

\section{Determinaciones en la vegetación}

En cada uno de los sitios se instalaron aleatoriamente y en sentido perpendicular al cauce principal tres transectas de $30 \mathrm{~m}$ cada una y se realizaron lecturas de puntos en línea (Levy and Madden 1933) con toques cada $20 \mathrm{~cm}$ (150 puntos por transecta). En cada toque se registró la presencia de las siguientes variables: vegetación por especie, suelo desnudo, mantillo y muerto en pie (Suárez et al. 2010). Además, alrededor de cada transecta se realizaron dos cortes manuales del pastizal con marco de $0.1 \mathrm{~m}^{2}$. En gabinete, la vegetación se determinó en especies con valor forrajero principal y especies indicadoras de deterioro (se consideró planta indicadora aquella cuya presencia señala una pérdida en la capacidad productiva del pastizal [Bonvisutto et al. 2008; Suárez et al. 2010]), lo cual difiere del criterio ecológico que considera una pérdida en la biodiversidad original de los pastizales). Luego, a partir de los valores totales de las transectas por sitio se calculó la cobertura aérea absoluta de cada variable mediante la fórmula: presencia de toque/presencia total de toques (150) x 100\%. El forraje cosechado en los marcos fue secado en estufa a $60^{\circ} \mathrm{C}$ hasta peso constante, para determinar la biomasa aérea disponible del pastizal (kg MS/ha).

\section{Determinaciones físicas y químicas del suelo}

En cada sitio se realizaron tres mediciones de resistencia mecánica $(\mathrm{RM})$ del suelo a $5 \mathrm{~cm}$ de profundidad con un penetrómetro de cono de acción vertical descripto en Zerpa et al. (2013). Se recolectaron tres muestras de los primeros 5 $\mathrm{cm}$ de suelo con un cilindro metálico biselado de $100 \mathrm{~cm}^{3}$; en laboratorio, estas muestras se secaron en estufa a $60^{\circ} \mathrm{C}$ para determinar el 
peso seco y la densidad aparente (Dap) con el método del cilindro (Gabriels and Lobo 2002), usando la fórmula

$$
\operatorname{Dap}\left(\mathrm{g} / \mathrm{cm}^{3}\right)=\text { peso seco }(\mathrm{g}) \times 100 / 100 \mathrm{~cm}^{3}
$$

Además, se recolectaron por sitio tres muestras de suelo con características físicoquímicas iniciales descriptas por Utrilla et al. (2014a) (a razón de una muestra compuesta por transecta) entre 0 y $15 \mathrm{~cm}$ y tres muestras adicionales sin vegetación en el sector seco del mallín con mayor degradación. Luego, en laboratorio se determinaron los siguientes parámetros: $\mathrm{pH}$ en pasta saturada, con potenciómetro, resistencia eléctrica (RE) en pasta, con conductímetro (Dewis and Freitas 1984), carbono orgánico (CO) mediante digestión con solución sulfocrómica y posterior colorimetría (Heanet 1984) y materia orgánica $(\mathrm{MO}=\mathrm{CO} 1.728)$.

\section{Determinaciones químicas del agua freática}

En el sector húmedo y las tres condiciones del mallín, se instalaron tres freatímetros (uno por transecta de vegetación) de PVC, perforados a $1.5 \mathrm{~m}$ de profundidad para recolectar tres muestras de agua freática con un vaso de precipitado de $50 \mathrm{~mL}$ y determinar en laboratorio el $\mathrm{pH}$, la conductividad eléctrica (CE) (Standard Methods for the Examination of Water and Wastewater 2005), las concentraciones de cloruro (según norma SM 4500-CL-B), calcio total y disuelto, y magnesio total y disuelto (según norma SM 2340 C), potasio total, sodio total (según norma SM 3500-Na B) y la relación de absorción de sodio (RAS) (Ayers and Westcot 1987). A partir de la CE y RAS del agua se evaluaron, respectivamente, los riesgos de salinización y sodificación de acuerdo con categorías de calidad de agua según normas Riverside (Thorne and Peterson 1954).

\section{Determinación de la biomasa radical}

En los tres sitios del sector húmedo del mallín se recolectaron por fecha tres muestras de suelo con raíces de plantas completos de acuerdo al método de las púas (Bohm 1979) hasta los $15 \mathrm{~cm}$ de profundidad que representaron un volumen de $1.875 \mathrm{dm}^{3}$. Las muestras se procesaron en laboratorio en las siguientes etapas: a) subdivisión en sub-muestras a distancias regulares de $5 \mathrm{~cm}$ (determinado por la disposición de los clavos), lo cual resulta en $3 \mathrm{sub}$-muestras por pan de suelo que corresponden a 3 profundidades de interés (0-5, 5-10 y 10-15 cm); b) lavado de las sub-muestras y separación de las raíces, y c) secado en estufa a $60^{\circ} \mathrm{C}$ hasta peso constante y pesado con balanza analítica de precisión para determinar la biomasa radical por profundidad y volumen $\left(0.625 \mathrm{dm}^{3}\right)$, y total mediante la sumatoria de las tres profundidades descriptas y el volumen total $\left(1.875 \mathrm{dm}^{3}\right)$.

\section{Diseño y análisis estadístico}

Se exploró la variabilidad entre condiciones y sectores en las 2 fechas anuales y los 3 años del estudio, mediante análisis de componentes principales (ACP) y matrices de correlación (Orloci 1978). Para ello se consideraron las siguientes variables de la vegetación y el suelo: especies indicadoras, suelo desnudo, mantillo, biomasa aérea disponible, RM, Dap, pH, RE y MO. A su vez, los valores de las variables descriptas se analizaron mediante una prueba tri-factorial $2 \times 2 \times 3$ (i.e., sector [S, factor fijo; Húmedo y Seco], fecha [F, factor fijo; diciembre y abril] y condición [C, factor fijo; Buena, Moderadamente Degradada y Muy Degradada]). La prueba se incluyó en un diseño completamente aleatorizado con tres repeticiones (resultantes de tres años) con arreglo en parcelas subdivididas (López Bautista and González Ramírez 2013), considerándose el S, F y C como tratamiento principal, tratamiento secundario y subtratamiento, respectivamente. Las unidades de estudio resultantes de cada sector, fecha y condición, fueron caracterizadas a partir del valor promediodelasmediciones de vegetación y suelo realizadas, respectivamente, en tres transectas y tres muestras (seudorepeticiones) para estimar mejor las variables de respuesta. Los resultados se sometieron a un análisis de la varianza (ANVA) con el procedimiento PROC GLM (SAS 2002-2008) de acuerdo con el siguiente modelo:

$$
\begin{aligned}
& \quad \mathrm{Y}_{\mathrm{ijkl}}=\mu+\alpha_{\mathrm{i}}+\varepsilon(\mathrm{a})_{\mathrm{il}}+\beta_{\mathrm{j}}+\varepsilon(\mathrm{b})_{\mathrm{ij}}+\gamma_{\mathrm{k}}+\gamma \beta_{\mathrm{jk}}+\gamma \alpha_{\mathrm{ik}} \\
& +\alpha \beta_{\mathrm{ij}}+\varepsilon_{\mathrm{ijk}}
\end{aligned}
$$

donde

$Y_{\mathrm{ijk} 1}=$ variable respuesta medida en la ijkésima unidad de estudio

$\mu=$ media general

$\alpha_{i}=$ efecto del i-ésimo sector

$\varepsilon(\mathrm{a})_{\mathrm{il}}=$ error asociado a la parcela grande

$\beta_{\mathrm{j}}=$ efecto de la j-ésima fecha

$\varepsilon(\mathrm{b})_{\mathrm{ij}}=$ error asociado a la parcela pequeña 
$\gamma_{\mathrm{k}}=$ efecto de la k-ésima condición

$\gamma \beta_{\mathrm{jk}}=$ efecto de la interacción entre la k-ésima condición y la j-ésima fecha

$\gamma \alpha_{\mathrm{ik}}=$ efecto de la interacción entre la k-ésima condición y el i-ésimo sector

$\alpha \beta_{\mathrm{ij}}=$ efecto de la interacción entre el i-ésimo sector y la j-ésima fecha

$$
\varepsilon_{\mathrm{ijkl}}=\text { error aleatorio }
$$

Los valores de CE y de RAS del agua freática se incluyeron en un diseño completamente aleatorizado con tres repeticiones (resultantes de tres años). Las unidades de estudio provenientes de cada condición de degradación se caracterizaron según el valor promedio de los registros realizados en tres freatímetros (seudorepeticiones) para estimar mejor los parámetros evaluados. Los resultados se sometieron a un análisis de la varianza (ANVA) con el procedimiento PROC GLM (SAS 2002-2008) de acuerdo al siguiente modelo:

$Y_{i j}=\mu+\alpha_{i}+\varepsilon_{i j}$

donde

$Y_{i j}=$ variable respuesta medida en la ij-ésima unidad de estudio

$\mu=$ media general

$\alpha_{i}=$ efecto de la i-ésima condición

$\varepsilon_{\mathrm{ij}}=$ error experimental asociado a la ij-ésima unidad de estudio

Los valores de biomasa radical se analizaron mediante una prueba bi-factorial 3x3 (i.e., condición de degradación [C, factor fijo; Buena, Moderadamente Degradada y Muy Degradada] y Profundidad [P, factor fijo; 05, 5-10 y 10-15 cm]). La prueba se incluyó en un diseño completamente aleatorizado con 6 repeticiones (resultantes de 3 muestras y dos fechas) y las unidades de estudio provenientes de cada condición y profundidad se sometieron al ANVA con el procedimiento PROC GLM (SAS 2002-2008) según el siguiente modelo:

$$
Y_{i j k}=\mu+\alpha_{i}+\beta_{j}+\alpha \beta_{i j}+\varepsilon_{i j}
$$

donde

$Y_{i j}=$ variable respuesta medida en la ijk-ésima unidad de estudio

$\mu=$ media general $\alpha_{i}=$ efecto de la i-ésima condición

$\beta_{\mathrm{j}}=$ efecto de la j-ésima profundidad

$\alpha \beta_{\mathrm{ij}}=$ efecto de la interacción entre la i-ésima condición y la j-ésima profundidad

$\varepsilon_{\mathrm{ijk}}=$ error experimental asociado a la ijkésima unidad de estudio

Los supuestos del ANVA de distribución normal y varianzas homogéneas se probaron con el PROC UNIVARIATE y PROC GLM de los residuales (SAS 2002-2008), respectivamente. En el caso de la RAS se debió analizar la variable transformada (raíz cuadrada) para cumplir con los supuestos de normalidad y varianzas homogéneas, y con éste último para Dap. Además, se analizó la biomasa aérea disponible, la RE y la biomasa radical transformada $\left(\operatorname{logaritmo}_{10}\right.$ ) para cumplir con los mismos supuestos. Las medias de las variables de vegetación, suelo y agua se compararon con la prueba de Duncan (significancia $=1 \%$ ) y las diferencias entre las medias de las interacciones y de la variable biomasa radical se ajustaron mediante la prueba Tukey-Kramer (significancia=5\%). Por último, los valores de RM y Dap del suelo se sometieron a un análisis de regresión (PROGREG.SAS) para determinar su relación con la humedad edáfica mediante el método gravimétrico descripto en: https://tinyurl.com/ y5k9w8ak.

\section{Resultados}

\section{Análisis de componentes principales}

El análisis detectó tres grupos de sitios (Figura 1): Grupo 1 (G1) [Bueno-Húmedo y Moderadamente Deteriorado-Húmedo]; Grupo 2 (G2) [Moderadamente DeterioradoSeco] y Grupo 3 (G3) [Muy DeterioradoHúmedo y Muy Deteriorado-Seco]. En este contexto, el G1 estuvo asociado a la MO y la biomasa aérea disponible y el G2 se asoció con la RE del suelo (Figura 1). En cambio, el G3 se correlaciono principalmente con especies indicadoras, suelo desnudo, mantillo y Dap (Figura 1). Asimismo, los componentes 1 y 2 (CP1 y CP2) del análisis explicaron en conjunto el $75 \%$ de la variabilidad total entre condiciones y sectores del mallín. En función de ello, el CP1 explico un 55.8\% de la variación y separó los G1 y G2 del G3. A su vez, el CP2, con un $19.2 \%$ de la variación, separó el G1 del G2 (Figura 1). 


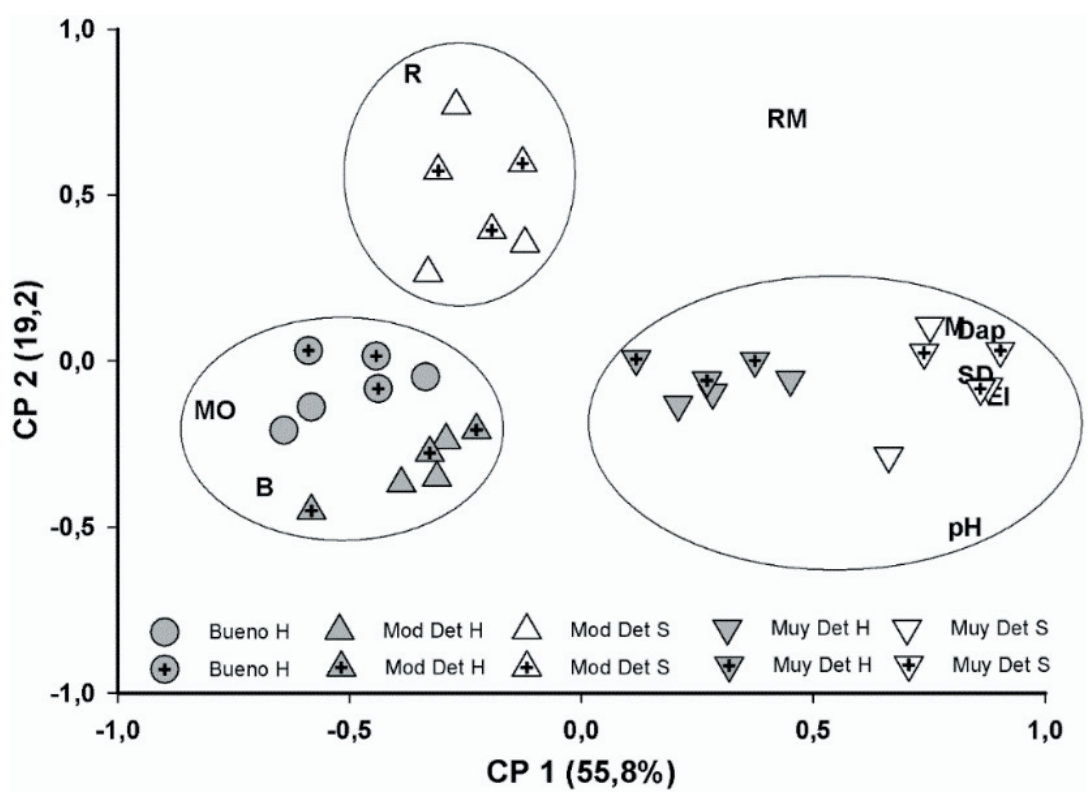

Figura 1. Análisis de componentes principales de las variables especies indicadoras (EI), suelo desnudo (SD), mantillo (M), biomasa aérea disponible (B), resistencia mecánica (RM), densidad aparente (Dap), pH, resistencia eléctrica (R) y materia orgánica $(\mathrm{MO})$ para cada condición y sector del mallín. Los símbolos indican la condición del mallín: Bueno $(\square)$; Moderadamente Deteriorado $(\Delta)$ y Muy Deteriorado $(\nabla)$. Los símbolos sombreados y blancos indican el sector Húmedo y Seco, respectivamente. Finalmente, los símbolos sin y con cruces corresponden a diciembre y abril, respectivamente. Mod: Moderadamente; Det: Deteriorado; H: Húmedo; S: Seco.

Figure 1. Principal component analysis of variables indicator species (IS), bare soil (BS), litter (L), available aerial biomass (AAB), mechanical resistance (MR), bulk density (BD), pH, electric resistance (R) and organic matter (OM) for each condition and sector of meadow. The symbols indicate the condition of meadow: Good ( $\boldsymbol{Q}$ ); Moderate Degraded $(\Delta)$ and Highly Degraded $(\nabla)$. Shaded and white symbols indicate Wet and Dry sector, respectively. Finally, the symbols without and with crosses correspond to December and April, respectively. Mod: Moderately; Det: Deteriorated; H: Wet; S: Dry.

\section{Variables de vegetación}

Las especies con valor forrajero principal (EF) ocuparon entre el 50 y $65 \%$ y entre el 15 y $25 \%$ en los sitios húmedo y seco muy degradados, respectivamente, con predominio de Poa pratensis y Juncus balticus (10-35\%) en el primer sitio y de J. balticus (10-15\%) en el sitio seco. En cambio, en los sitios H Buena y H Mod Deg, y S Mod Deg, las EF superaron el 70\%, destacándose, respectivamente, Carex gallana (65-95\%), Agrostis sp. (>35\%) y Poa pratensis (25-35\%) con Festuca pallescens (15-35\%).

A partir del ANVA realizado, la cobertura de especies indicadoras (EI) manifestó una media general de $15 \%$ y estuvo influenciada por el Sector (S), Fecha (F) y Condición (C). En este contexto, la media de EI en el sector seco $(23.2 \mathrm{a} \%)$ fue mayor al sector húmedo $(9.5 \mathrm{~b} \%)$ y el valor reportado en diciembre $(19.1 \mathrm{a} \%)$ se destacó sobre la media de abril $(10.9 \mathrm{~b} \%)$. Además, el valor del parámetro bajo condición muy degradada (31.9a\%) superó a las condiciones de degradación moderada $(5.3 \mathrm{~b} \%)$ y buena $(0.6 \mathrm{~b} \%)$. A su vez, el análisis detectó interacciones SxF, CxF y CxS (Tabla 1). Por ello, según la interacción CxS de interés reportada, las medias de EI en los sitios H Muy Deg y S Muy Deg del mallín (próximas a 25 y $40 \%$, respectivamente) superaron (6 veces) a los valores de los sitios H Mod Deg y H Buena y S Mod Deg ( $<10 \%)$ (Tabla 1$)$. En cambio, no hubo diferencias $(P>0.05)$ entre las medias de los sitios H Buena y H Mod Deg (Tabla 1). Cabe mencionar que, las EI estuvieron representadas principalmente por Taraxacum officinale (Diente de león) y Stipa chrysophylla (Coirón amargo) con valores máximos del 30-35\% y 40-50\% en diciembre en los sitios húmedo y seco bajo condición muy degradada, respectivamente. Asimismo, otras EI relevadas fueron Acaena magellánica e Hipochaeris hookeri con coberturas menores a 3 y $6 \%$, respectivamente.

El análisis de la cobertura de suelo desnudo (SD) con una media general de $4 \%$, detectó efectos del Sector y Condición sobre el parámetro. En función de ello, la media del SD en el sector seco (8.3a\%) fue mayor al valor informado en el sector húmedo $(1.1 \mathrm{~b} \%)$ y la media bajo condición muy degradada $(9.6 \mathrm{a} \%)$ fue superior a los valores bajo las condiciones de degradación moderada $(0.1 \mathrm{~b} \%)$ y buena $(0.4 b \%)$. A su vez, de acuerdo a la interacción 
CxS detectada, el valor del SD en el sitio S Muy Deg del mallín (cercano a 15\%) superó (30 veces) a la media del sitio S Mod Deg (<1\%) (Tabla 1). En cambio, no se reportó diferencias $(P>0.05)$ entre los tres sitios húmedos. (Tabla 1).

La cobertura del mantillo (M) (media general: $7.5 \%$ ) estuvo afectada por el Sector y Condición. En virtud de ello, la media del M en el sector seco (11.9a\%) fue mayor respecto al sector húmedo $(4.6 \mathrm{~b} \%)$ y el valor bajo condición muy degradada (14.0a\%) superó a las medias bajo condiciones de degradación moderada $(3.4 \mathrm{~b} \%$ ) y buena $(2.9 \mathrm{~b} \%)$. Asimismo, el análisis no detectó interacciones $(P>0.05)$ entre los factores descriptos. En este contexto, la media del M en el sitio S Muy Deg (próximo a $20 \%$ ) superó entre 6 y 18 veces a los valores de los sitios restantes ( $<10 \%)$, a excepción del valor informado en el sitio H Muy Deg (Tabla 1).

Cabe mencionar que, en ambos sectores y fechas y todas las condiciones de degradación el material vegetal muerto en pie relevado en los sitios de estudio fue inferior a $10 \%$.

El Sector y la Condición afectaron la biomasa aérea disponible (B) del pastizal (media general: $2000 \mathrm{~kg}$ MS/ha). En este contexto, la media transformada $\left(\log _{10}\right)$ del parámetro en el sector húmedo (7.6a, $2687 \mathrm{~kg}$ MS/ha) se destacó sobre el valor reportado en el sector seco (6.5b, $976 \mathrm{~kg}$ MS/ha) y las medias del $\mathrm{Log}_{10}$ bajo condición buena (7.8a, $2739 \mathrm{~kg}$ MS/ha) y moderada (7.9a, $3023 \mathrm{~kg}$ MS/ha) superaron al valor bajo condición muy degradada (6.2b, 613 kg MS/ha). A su vez, el análisis no detectó interacciones $(P>0.05)$ entre los factores evaluados. En virtud de ello, las medias del $\log _{10}$ del parámetro en los sitios $\mathrm{H}$ Buena y $\mathrm{H}$ Mod Deg fueron superiores, respectivamente, a los valores reportados en los sitios H Muy Deg (3 y 5 veces) y S Muy Deg (8.5 y 14.0 veces) del mallín (Tabla 1).

\section{Variables físicas y químicas del suelo}

En virtud de la relación entre la RM y la Dap con la humedad edáfica, se corrigieron con esta variable los valores de ambos parámetros mediante análisis de regresión. En este contexto, la RM del suelo (media general: 6.4 MPa) estuvo influenciada por el Sector y la Condición. Por ello, la media del parámetro en el sector seco (8.4a $\mathrm{MPa}$ ) supero al valor del sector húmedo (5.1b MPa) y las medias bajo condición muy degradada (7.2a MPa) y moderada (6.9a MPa) fueron mayores respecto a la condición buena (4.0b MPa). Además, el análisis detectó interacción CxS, con lo cual la media de la RM en el sitio H Muy Deg fue superior (1.6 veces) al valor del H Buena. En cambio, en los sitios húmedo y seco no hubo

Tabla 1. Medias de especies indicadoras (\%), suelo desnudo(\%), mantillo (\%), biomasa aérea disponible (kg MS/ha), resistencia mecánica $(\mathrm{MPa})$, densidad aparente $\left(\mathrm{g} / \mathrm{cm}^{3}\right), \mathrm{pH}$, resistencia eléctrica $(\mathrm{S} . \mathrm{cm})$ y materia orgánica $(\%)$ por sector del mallín y condición. ${ }^{1}$ Error estándar de la media; ${ }^{2}$ Sig.: significancia, ${ }^{+}: P<0.01$ para Sector $(\mathrm{S})$, Fecha ( F) y Condición $(\mathrm{C})$; ${ }^{3}$ Mod: Moderadamente; Deg: Degradado; disp.: disponible; *: Logaritmo de la media; ${ }^{+}$Raíz cuadrada de la media. Letras distintas indican diferencias significativas $(P<0.05)$ entre condiciones para cada parámetro.

Table 1. Means of indicator species (\%), bare soil (\%), litter (\%), available aerial biomass ( $\mathrm{kg} \mathrm{DM} / \mathrm{ha})$, mechanical resistance $(\mathrm{MPa})$, bulk density $\left(\mathrm{g} / \mathrm{cm}^{3}\right)$, $\mathrm{pH}$, electric resistance $(\mathrm{S} . \mathrm{cm})$ and organic matter (\%) by sector of meadow and condition. ${ }^{1}$ Mean standard error; ${ }^{2}$ Sig.: significant, ${ }^{+}: P<0.01$ for Sector $(\mathrm{S})$, Date $(\mathrm{F})$ and Condition $(\mathrm{C}){ }^{3}{ }^{3}$ Mod: Moderately; Deg: Degraded; disp.: available; ${ }^{*}$ : Mean logarithm; ${ }^{+}$: Mean square root. Different letters indicate significant differences $(P<0.05)$ between conditions for each parameter.

\begin{tabular}{|c|c|c|c|c|c|c|c|}
\hline \multirow{2}{*}{$\begin{array}{l}\text { Sector del mallín } \\
\text { Condición }^{3}\end{array}$} & \multicolumn{3}{|c|}{ Húmedo } & \multicolumn{2}{|c|}{ Seco } & \multirow[t]{2}{*}{$\mathrm{EEM}^{1}$} & \multirow[t]{2}{*}{ Sig. $^{2} P \triangleleft 0.01^{+} P<0.05$} \\
\hline & Buena & Mod. Deg. & Muy Deg. & Mod.Deg. & Muy Deg. & & \\
\hline Especies indicadoras & $0.6 \mathrm{c}$ & $3.9 \mathrm{c}$ & $24.0 \mathrm{~b}$ & $6.7 \mathrm{c}$ & $39.7 \mathrm{a}$ & 0.7 & $\mathrm{~S}^{+} \mathrm{F}^{+} \mathrm{C}^{+} \mathrm{SxF} \mathrm{CxF} \mathrm{CxS}$ \\
\hline Suelo desnudo & $0.4 \mathrm{~b}$ & $0.0 \mathrm{~b}$ & $2.9 \mathrm{~b}$ & $0.2 b$ & $16.3 a$ & 0.3 & $\mathrm{~S}^{+} \mathrm{C}^{\dagger} \mathrm{CXS}$ \\
\hline Mantillo & $2.9 \mathrm{~b}$ & $1.1 \mathrm{~b}$ & 9.9ab & $5.6 b$ & $18.2 \mathrm{a}$ & 0.6 & $\mathrm{~S}^{+} \mathrm{C}^{+}$ \\
\hline Biomasa área disp. & $2739\left(7.9 a b^{*}\right)$ & $4418\left(8.3 a^{*}\right)$ & $903\left(6.7 c^{*}\right)$ & $1629\left(7.3 b c^{*}\right)$ & $323\left(5.7 d^{*}\right)$ & 171 & $\mathrm{~S}^{\dagger} \mathrm{C}^{\dagger}$ \\
\hline Resistencia mecánica & $4.0 \mathrm{~d}$ & $4.9 \mathrm{~cd}$ & $6.4 \mathrm{bc}$ & $8.9 a$ & 8.0ab & 0.1 & $\mathrm{~S}^{\dagger} \mathrm{C}^{\dagger} \mathrm{CxS}$ \\
\hline Densidad aparente & $0.18\left(0.42 \mathrm{~d}^{+}\right)$ & $0.42\left(0.64 c^{+}\right)$ & $0.77\left(0.87 a b^{+}\right)$ & $0.57\left(0.75 b c^{+}\right)$ & $0.85\left(0.92 \mathrm{a}^{+}\right)$ & 0.01 & $\mathrm{~S}^{\dagger} \mathrm{C}^{+}$ \\
\hline $\mathrm{pH}$ & $5.8 \mathrm{~d}$ & $6.6 \mathrm{c}$ & $7.2 b$ & $5.3 \mathrm{~d}$ & $8.0 \mathrm{a}$ & 0.03 & $\mathrm{C}^{+} \mathrm{CxS}$ \\
\hline Resistencia eléctrica & $1364\left(7.2 b^{*}\right)$ & $430\left(6.0 \mathrm{~d}^{*}\right)$ & $818\left(6.7 c^{*}\right)$ & $2404\left(7.8 a^{*}\right)$ & $673\left(6.5 c^{*}\right)$ & 37 & $\mathrm{~S}^{+} \mathrm{C}^{+} \mathrm{CxS}$ \\
\hline Materia orgánica & $11.9 \mathrm{a}$ & $11.5 \mathrm{a}$ & $7.7 \mathrm{bc}$ & $10.2 \mathrm{ab}$ & 7.1c & 0.2 & $\mathrm{~S}^{+} \mathrm{C}^{+}$ \\
\hline
\end{tabular}


diferencias $(P>0.05)$ entre las condiciones moderada y muy degradada (Tabla 1 ).

La Dap (media general: $0.56 \mathrm{~g} / \mathrm{cm}^{3}$ ) estuvo afectada por elSectory la Condición. En función de ello, el valor de la RCuad del parámetro en el sector seco $\left(0.83 \mathrm{a}, 0.71 \mathrm{~g} / \mathrm{cm}^{3}\right)$ fue mayor a la media del sector húmedo $\left(0.65 \mathrm{~b}, 0.46 \mathrm{~g} / \mathrm{cm}^{3}\right)$ y hubo diferencias entre condiciones a favor de la condición muy degradada $(0.90 \mathrm{a}, 0.81$ $\mathrm{g} / \mathrm{cm}^{3}$ ) respecto a las condiciones moderada $\left(0.70 \mathrm{~b}, 0.49 \mathrm{~g} / \mathrm{cm}^{3}\right)$ y buena $\left(0.42 \mathrm{c}, 0.18 \mathrm{~g} / \mathrm{cm}^{3}\right)$. En correspondencia con ello, la Dap aumento, respectivamente, desde 1.5 hasta 4.5 veces en el mallín con mayor deterioro en relación a aquél bajo condición moderada y buena. A su vez, en el análisis no se detectó $(P>0.05)$ interacción CxS y las medias de Dap en los sitios S Muy Deg y H Muy Deg superaron (desde 2 hasta 5 veces) a los valores de los sitios H Mod Deg y H Buena. (Tabla 1).

El pH del suelo (media general: 6.6) estuvo influenciado por la Condición y el análisis detectó diferencias entre condiciones con valores que variaron desde moderadamente ácido (escala: 5.5-6.0) bajo condición buena (5.8b) y moderada (5.9b) a moderadamente alcalino (escala: 7.5-8.0) bajo condición muy degradada (7.6a). A partir de la interacción CxS detectada, los valores del $\mathrm{pH}$ variaron entre condiciones en forma contrastante según el sector del mallín. En este contexto, en el sector húmedo el parámetro varió desde moderadamente ácido (condición buena) a débilmente alcalino (condición muy degradada). En cambio, en el sector seco el pH osciló desde netamente ácido (condición moderada) a moderadamente alcalino (condición muy degradada) (Tabla 1).

El Sector y la Condición afectaron la RE edáfica (media general: 1138 S.cm). En virtud de ello, la media del $\log _{10}$ en el sector seco (7.1a, 1539 S.cm) superó al valor del sector húmedo $(6.6 \mathrm{~b}, 871 \mathrm{~S} . \mathrm{cm})$ y la RE bajo condición buena (7.2a, 1364 S.cm) fue mayor respecto a la condición muy degradada (6.6b, $746 \mathrm{~S} . \mathrm{cm})$. Cabe mencionar que los valores de RE $>1000$ y $<1000$ S.cm equivalen a nula y moderados problemas de salinidad, respectivamente. A su vez, el análisis detectó interacción CxS, con lo cual se obtuvo una respuesta contrastante en la variación del parámetro entre condiciones según el sector del mallín. Por lo tanto, el valor del $\log _{10}$ de RE del sitio $H$ Buena superó, respectivamente, (3.0 y 1.5 veces) a las medias del parámetro de los sitios H Mod Deg $(\mathrm{RE}<500$ S.cm, equivalente a fuertes problemas de salinidad) y H Muy Deg. Además, el valor del sitio S Mod Deg fue mayor (3.5 veces) respecto al S Muy Deg (Tabla 1).

El Sector y la Condición influenciaron el contenido de $\mathrm{MO}$ del suelo (media general: $9.7 \%$ ). Por ello, el valor de MO del sector húmedo (10.4a) fue mayor respecto al sector seco $(8.7 b)$ y las medias del parámetro bajo condición buena (11.9a) y moderada (10.9a) superaron al valor bajo condición muy degradada (7.4b). Sin embargo, todos los valores informados fueron altos (escala: $7-12 \%)$ (Tabla 1$)$, y no se detectó $(P>0.05)$ interacción CxS. En este contexto, las medias de MO de los sitios H Buena y H Mod Deg fueron mayores (1.5 veces) a los valores de los sitios húmedo y seco bajo condición muy degradada (Tabla 1).

\section{Variables químicas del agua freática}

Los valores del $\mathrm{pH}$ (media desvío estándar) del agua freática variaron desde ligeramente ácido $(6.4 \pm 0.1)$ bajo condición buena del mallín

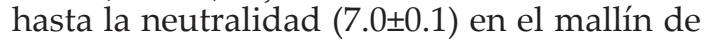
condición muy degradada. El análisis no detectó efecto $(P>0.05)$ de la Condición sobre la $C E$ del agua freática. Por lo tanto, no hubo diferencias $(P>0.05)$ entre los valores reportados del parámetro bajo condición buena (561a $\mu \mathrm{S} /$ $\mathrm{cm})$, moderada (700a $\mu \mathrm{S} / \mathrm{cm}$ ) y muy degradada (675a $\mu \mathrm{S} / \mathrm{cm})$ de los mallines. Además, los cloruros (media \pm desvío estándar) variaron desde $58.2 \pm 45.2 \mathrm{mg} / \mathrm{L}$ (condición buena) hasta $109.8 \pm 3.2 \mathrm{mg} / \mathrm{L}$ (condición moderada). La RAS estuvo influenciada por la Condición y se informó un mayor valor de RCuad de RAS bajo la condición moderada $(1.94 a, 3.91)$ respecto a la condición buena $(1.13 \mathrm{c}, 1.29) \mathrm{y}$ ausencia de diferencias entre las condiciones moderada y muy degradada $(1.67 a b, 2.83)$. En concordancia con lo descripto, la concentración de sodio total (media \pm desvío estándar) varió desde $48.0 \pm 12.8 \mathrm{mg} / \mathrm{L}$ (condición buena) hasta $151.7 \pm 77.2 \mathrm{mg} / \mathrm{L}$ (condición moderada), con un valor intermedio de $106.2 \pm 37.4 \mathrm{mg} / \mathrm{L}$ (condición muy degradada).

\section{Biomasa radical}

El análisis del modelo de estudio detectó efecto de la Condición (C) y la Profundidad (P) edáfica del mallín sobre la biomasa radical (BR) (media general: $54.0 \mathrm{~g} / \mathrm{dm}^{3}$ ) (Tabla 2). En este contexto, la media del $\log 10$ de la BR bajo condición buena $\left(4.6 \mathrm{a}, 100.4 \mathrm{~g} / \mathrm{dm}^{3}\right)$ fue mayor en relación a los valores bajo condición moderada $\left(3.4 \mathrm{~b}, 36.6 \mathrm{~g} / \mathrm{dm}^{3}\right)$ y muy degradada 
Tabla 2. Medias de biomasa radical $\left(\mathrm{g} / \mathrm{dm}^{3}\right)$ por condición y profundidad del suelo ( $\mathrm{cm}$ ) del mallín. ${ }^{1}$ Mod.: Moderadamente; Deg: Degradado; ${ }^{*}$ : Logaritmo de la media; ${ }^{2}$ Error estándar de la media. ${ }^{3}$ C: Condición, P: Profundidad. Letras distintas indican diferencias significativas $(P<0.05)$ entre medias para cada condición y profundidad.

Table 2. Means of radical biomass $\left(\mathrm{g} / \mathrm{dm}^{3}\right)$ by condition and soil depth (cm) of meadow. ${ }^{1}$ Mod.: Moderately; Deg: Degraded; *: Mean logarithm; ${ }^{2}$ Mean Standard Error. ${ }^{3}$ C: Condition, P: Soil Depth. Different letters indicate significant differences $(P<0.05)$ between means for each condition and soil depth.

\begin{tabular}{lccccc}
\hline Condición del mallín'/Profundidad & Buena & $\begin{array}{c}\text { Mod. } \\
\text { Deg. }\end{array}$ & $\begin{array}{c}\text { Muy } \\
\text { Deg. }\end{array}$ & $\begin{array}{c}\text { EEM }^{2} \\
\text { Significancia } \\
P<0.05\end{array}$ \\
\hline $0-5$ & $79.3\left(4.4 \mathrm{a}^{*}\right)$ & $56.7\left(4.0 \mathrm{ab}^{*}\right)$ & $42.6\left(3.7 \mathrm{~b}^{*}\right)$ & 1.7 & C P CxP \\
$5-10$ & $112.2\left(4.7 \mathrm{a}^{*}\right)$ & $33.6\left(3.5 \mathrm{bc}^{*}\right)$ & $22.8\left(3.1 \mathrm{c}^{*}\right)$ & & \\
$10-15$ & $109.6\left(4.7 \mathrm{a}^{*}\right)$ & $19.5\left(2.9 \mathrm{c}^{*}\right)$ & $9.4\left(2.2 \mathrm{~d}^{*}\right)$ & & \\
\hline
\end{tabular}

$\left(3.0 \mathrm{c}, 24.9 \mathrm{~g} / \mathrm{dm}^{3}\right)$. A su vez, éstos últimos difirieron entre sí a favor de la condición moderada. Asimismo, el valor del Log10 de BR a la profundidad 0-5 cm $\left(4.0 \mathrm{a}, 59.5 \mathrm{~g} / \mathrm{dm}^{3}\right)$ superó a las medias de las profundidades 5$10 \mathrm{~cm}\left(3.8 \mathrm{~b}, 56.2 \mathrm{~g} / \mathrm{dm}^{3}\right)$ y $10-15 \mathrm{~cm}(3.2 \mathrm{c}, 46.2$ $\left.\mathrm{g} / \mathrm{dm}^{3}\right)$, y la media de esta última estuvo por debajo respecto a la profundidad intermedia. A su vez, el análisis detectó interacción CxP, con lo cual las medias transformadas de la BR bajo condición buena fueron mayores respecto a los valores del parámetro bajo condición moderada a las profundidades $5-10$ y $10-15 \mathrm{~cm}$ y muy degradada a todas las profundidades (Tabla 2). En correspondencia con esto último, la BR se redujo desde 2 hasta 11 veces entre las condiciones buena y muy degradada del mallín a las profundidades 0-5 y $10-15 \mathrm{~cm}$, respectivamente. Además, hubo una respuesta contrastante en la variación de la BR con la profundidad según la condición. En virtud de ello, la BR no se diferenció $(P>0.05)$ con la profundidad bajo condición buena, y en general el parámetro disminuyó a mayor profundidad bajo condición moderada (entre $0-5$ y $10-15 \mathrm{~cm}$ ) y muy degradada (entre todas las profundidades) (Tabla 2). Finalmente, la BR Total (media \pm desvío estándar) bajo condición buena $\left(100.4 \pm 15.0 \mathrm{~g} / \mathrm{dm}^{3}\right)$ se redujo 2.7 y 4.0 veces respecto a las condiciones moderada $\left(36.6 \pm 8.1 \mathrm{~g} / \mathrm{dm}^{3}\right)$ y muy degradada $(24.9 \pm$ $4.1 \mathrm{~g} / \mathrm{dm}^{3}$ ) del mallín, respectivamente.

\section{Discusión}

\section{Análisis de componentes principales}

El análisis integral de la información permitió asociar las principales variables que describen el mayor deterioro de los sitios de estudio. En este contexto, la cobertura de especies indicadoras (EI), el suelo desnudo, el mantillo y la Dap representan las "variables de la vegetación y el suelo" asociados con el deterioro avanzado de los sitios húmedo y seco, lo cual se corresponde con los hallazgos de Bonvisutto et al. (2008) en mallines secos de condición pobre del NO de Río Negro y de Suárez et al. (2010) en mallines húmedos y secos muy degradados del Sur de Santa Cruz en los cuales reportaron un incremento de los parámetros vegetativos descriptos. Además, coincide con los resultados preliminares informados por Utrilla et al. (2012) en los sitios más degradados del presente estudio y en mallines húmedos y secos de Río Negro degradados por el sobrepastoreo que manifestaron un aumento en la densidad volumétrica edáfica (Enriquez et al. 2015). Por el contrario, la biomasa aérea disponible (B) del pastizal y el contenido de $\mathrm{MO}$ edáfica, por un lado, y la RE del suelo, por el otro, se corresponden respectivamente con la condición buena y moderada de los sitios húmedos, y la condición de degradación moderada del sitio seco.

En este contexto, al comparar los resultados del ACP con aquéllos del modelo factorial analizado, podría afirmarse que en los sitios húmedos y secos del presente estudio la cobertura de EI representa un parámetro vegetativo de utilidad para describir la condición más degradada del mallín. A su vez, la Dap surge como un indicador edáfico recomendable para explicar la condición más deteriorada de este ambiente. En cambio, la B y el contenido de $\mathrm{MO}$ resultan en indicadores óptimos para caracterizar las condiciones buena y moderadamente degradada de los sitios húmedos del mallín.

\section{Variables de vegetación}

El deterioro avanzado de los sitios húmedo y seco del mallín estuvo representado principalmente por la mayor ocupación de especies indicadoras (EI). En favor de ello, este parámetro fue destacado preliminarmente por Utrilla et al. (2012) como una de las principales variables explicativas de las diferencias entre 
las condiciones más contrastantes del sitio húmedo del presente trabajo. En este contexto, la predominancia del Diente de león (referida como EI, en virtud que su presencia indica una pérdida en la capacidad productiva del pastizal) en este sitio se corresponde con las principales EI de deterioro relevadas por Suárez et al. (2010) y Vargas (2017) en mallines húmedos de valle y semihúmedos glaciares y fluviales del Sur de Santa Cruz, respectivamente. Además, Bonvisutto et al. (1992) informaron un aumento de esta especie con un mayor deterioro del pastizal de mallines periféricos del área de Sierras y Mesetas del NO de Patagonia y fue descripta (hasta 30\%) en la condición pobre de mallines secos de Pre-cordillera del NO de Río Negro (Bonvisutto 2008). De la misma manera, en Norte-América, Popolizio et al. (1994) reportaron un aumento de esta especie en un ecosistema húmedo similar (riparian ecosystem) bajo pastoreo continuo prolongado.

En cambio, en el sitio seco más degradado del mallín, la predominancia del Coirón amargo podría explicarse por los procesos de erosión hídrica desencadenados por sobrepastoreo (Collantes and Faggi 2003; Utrilla et al. 2014a) y que favorecerían el desecamiento del ambiente y el ingreso de especies xerófitas de la estepa (Bran et al. com. pers.). En concordancia con ello, Suárez et al. (2010) relevaron a esta especie entre las principales EI en los mallines secos de valle muy degradados del Sur de Santa Cruz. A su vez, en contraste con el aumento de EI en los sitios más degradados del presente estudio, se corresponde la disminución de especies con valor forrajero principal, sobre todo, en el sitio seco.

Con respecto a la cobertura de suelo desnudo (SD) informada en los sitios de estudio, fue citada preliminarmente por Utrilla et al. (2012) como parámetro destacado explicativo de las diferencias entre condiciones en los sitios del presente trabajo. Asimismo, el incremento del SD en el sitio seco más degradado coincide con el aumento del parámetro en los mallines secos de valle muy deteriorados descriptos por Suárez et al. (2010) y con los hallazgos de Bonvisutto et al. (2008) que reportaron hasta $25 \%$ de SD en mallines periféricos bajo condición pobre del NO de Río Negro.

En correspondencia con la disminución de la biomasa aérea disponible (B) del pastizal bajo la condición muy degradada del mallín respecto a las condiciones restantes, en mallines húmedos de Juncus balticus y secos de Festuca pallescens de Sierras y Mesetas del NO de Río Negro Bonvisutto et al. (2008) informaron bajo condición pobre, respectivamente, una reducción de cuatro y dos a tres veces y media en la producción anual de forraje respecto a la condición buena. A su vez, altas presiones de pastoreo prolongado disminuyeron la producción primaria neta aérea de la vegetación en mallines húmedos y secos del Norte de Río Negro (Enriquez et al. 2015) y en un mallín húmedo de Tierra del Fuego (Cipriotti et al. 2018). De la misma manera, en un ecosistema húmedo similar de Norte-América con predominio de Carex sp. la defoliación intensiva disminuyó la biomasa total (Clary 1995).

Asimismo, cabe mencionar que en términos relativos la mayor $B$ informada en el sitio húmedo bajo condición moderada del presente estudio respecto al sitio húmedo buena (Tabla 1), se explicaría por mayores temperaturas medias y lluvias mensuales en los mallines Las Horquetas en relación al mallín La Tapera, sobre todo en el período enero-marzo del año 2012, por un lado, y por el uso tardío del ganado en este período y año del sitio bajo condición moderada, por el otro (Utrilla et al. 2014a).

\section{Variables físicas y químicas del suelo}

Según Sellés et al. (2012), los valores de RM reportados en los sitios húmedo y seco fueron, respectivamente, muy altos y extremadamente altos. En este contexto, el mayor valor de RM en el sitio húmedo muy degradado en relación al sitio húmedo buena se corresponde con el mismo resultado informado por Utrilla et al. (2014b) en los mallines del presente estudio, lo cual indicaría una mayor compactación del suelo por efecto del pisoteo animal (Wilkins and Garwood 1986). En coincidencia con ello, en un ecosistema húmedo de NorteAmérica con predominio de Poa pratensis y Agrostis stolonífera, Clary (1995) registró un aumento en la RM a partir de un tratamiento de compactación impuesto.

Asimismo, los valores extremadamente altos de RM en los sitios secos podrían deberse al aumento de la densidad volumétrica y reducción de los espacios porosos (Wilkins and Garwood 1986) por el pisoteo animal que aumentaría la resistencia a la penetración resultante de una mayor compactación (Del Valle 1993). Además, reflejarían síntomas de erosión hídrica, tales como, plantas en pedestal y la presencia de surcos y cárcavas, 
bajo condiciones de degradación moderada y severa, respectivamente (Utrilla et al. 2014a). A su vez, la presencia de éstos síntomas en los sitios secos indicaría un estado avanzado de deterioro del mallín (Bonvisutto et al. 2008; Suárez et al. 2010; Bran et al. com. pers.).

En concordancia con lo descripto en los mallines más deteriorados, Radic et al. (2011) reportaron mayores valores de Dap en un mallín periférico de Tierra del Fuego con predominio de especies indicadoras de degradación. De la misma manera, en mallines húmedos del Norte de Río Negro y en humedales similares de Norte-América, Enriquez et al. (2015) y Kauffman and Krueger (1984) y Belsky et al. (1999), respectivamente, informaron un aumento de la densidad volumétrica por compactación del suelo a partir del pastoreo continuo intensivo del ganado.

Con respecto a las variables químicas del suelo, los fuertes problemas de salinidad del sitio húmedo con degradación moderada del presente estudio se corresponden con niveles altos de sales relevados por Lamoureux and Migliora (1999. Citado por Utrilla et al. 2014a) en suelos con drenaje impedido de mallines húmedos de la cuenca media del Río Coyle. A su vez, en casos extremos la presencia superficial de sales indicaría un deterioro severo del mallín, según Suárez et al. (2010), en virtud de la menor cobertura vegetal y aumento en la ocupación del suelo desnudo que favorecería la evaporación y por lo tanto el ascenso del agua por capilaridad y posterior salinización (Bonvisutto et al. 1992; Del Valle 1993) y fue reportada por Bonvisutto et al. (2008) en mallines húmedos (por disminución de la capacidad de infiltración) y secos bajo condición pobre de Sierras y Mesetas del NO de Patagonia.

Con relación al contenido de Materia Orgánica $(\mathrm{MO})$, la declinación del parámetro según el avance de la condición de degradación coincide con estudios en humedales de NorteAmérica sometidos a pastoreos intensivos (Belsky et al. 1999) y podría relacionarse con una menor aireación e infiltración hídrica, en virtud de la compactación del suelo por el pisoteo animal (Wilkins and Garwood 1986) relevada en los sitios con deterioro severo, y que estarían condicionando la descomposición de los restos vegetales de la MO. A su vez, el sobrepastoreo a largo plazo de estos ambientes reduce el contenido de carbono orgánico edáfico total, en virtud de una disminución en la biomasa aérea y subterránea de la vegetación (Enriquez et al. 2015), y en la acumulación del carbono orgánico particulado proveedor de la MO más lábil (Enriquez and Cremona 2018).

\section{Variables de calidad del agua freática}

Las medias de CE informadas bajo condición buena, moderada y muy degradada del mallín indican un bajo riesgo de salinización originada en el agua, con valores inferiores a $750 \mu \mathrm{S} / \mathrm{cm}$ que representa el umbral superior de la categoría de referencia C2 de Thorne and Peterson (1954). Sin embargo, bajo la condición muy degradada hubo un valor superior a $1000 \mu \mathrm{S} / \mathrm{cm}$, clasificándose como agua de tipo C3. En este contexto, cabe mencionar que en la C3 resulta crítica la existencia de buena permeabilidad edáfica para asegurar el drenaje de sales (Avellaneda et al. 2004), lo cual también resulta de la compactación del suelo por el pisoteo animal en condiciones húmedas bajo la condición más degradada del mallín. Sin embargo, dado los valores bajos de cloruros relevados en los sitios, no podría establecerse una relación directa entre la $C E$ del agua y aquéllos.

Los valores de la RAS reportados en todas las condiciones se encuentran por debajo de umbrales críticos para la supervivencia de la vegetación y por lo tanto se presenta un bajo riesgo de sodificación del suelo. En este sentido, para las categorías de riesgo de salinización con valores de CE inferiores a 750 $\mu \mathrm{S} / \mathrm{cm}$ el esquema tradicional de Riverside (Thorne and Peterson 1954) propone un límite superior de RAS entre 6 y 13, que supera a todos los valores del presente trabajo. Inclusive, para el valor de CE del sitio más degradado con riesgo salino categoría C3 la media de la RAS (3.75) fue inferior al límite superior del riesgo sódico que indica una RAS de 4-6. A pesar de ello, resulta evidente el aumento en la concentración de $\mathrm{Na}^{+}$reportada en los sitios bajo condición moderada y muy degradada respecto a la condición buena del presente estudio. Prueba de ello, Utrilla et al. (2014a) informaron valores preliminares mayores de sales totales, porcentaje de sodio y RAS ajustado en el sitio más degradado con relación a las condiciones restantes de este trabajo.

\section{Biomasa radical}

La reducción de la biomasa radical (BR) con la profundidad y la BR total en los sitios con 
deterioro moderado y severo respecto a la condición buena del mallín se explicaría por la mayor compactación edáfica por efecto del pisoteo animal (Wilkins and Garwood 1986) y elevada presión de pastoreo en condiciones húmedas (Del Valle 1993). Estos resultados se corresponden con otros trabajos que sostienen una disminución del crecimiento radicular con la compactación y altas presiones en el suelo, lo cual altera la capacidad de absorción de humedad en el perfil edáfico y reduce la disponibilidad de aire y con ello el intercambio gaseoso de las raíces con el suelo (Jensen 1994; Clary 1995; Roberson 1996; Flores 1999). Asimismo, las defoliaciones intensas en varias especies de gramíneas afectan el crecimiento y biomasa radicular (Caloin et al. 1990; Thornton and Millard 1996) y con alta carga animal se reduce el crecimiento aéreo y el peso radical en pasturas de raigrás perenne y trébol blanco (Curll and Wilkins 1983). De la misma manera, una presión alta de pastoreo en mallines del Norte de Patagonia redujo la biomasa radicular de la vegetación en virtud de una disminución en la biomasa aérea de aquélla (Enriquez et al. 2015). En correspondencia con estos trabajos, el mayor aporte de las especies indicadoras de degradación y por lo tanto menor ocupación de las especies con valor forrajero principal y menor biomasa aérea disponible del pastizal en el sitio muy degradado respecto al sitio bajo condición buena del presente estudio también podría explicar la disminución de la biomasa radical en el sitio con deterioro avanzado.

A su vez, la declinación de la biomasa radical con la profundidad del suelo en los sitios de condición moderada y muy degradados coincide con estudios en ecosistemas áridos del Monte Patagónico disturbados por el pastoreo (Rodríguez et al. 2006) y en pastizales tropicales de Centro-América (Jiménez Rodríguez and Arias Aguilar 2004) por una mayor compactación, entre otras características edáficas. Por el contrario, la variación no significativa de la biomasa radical con la profundidad relevada en el sitio bajo condición buena podría explicarse por la disponibilidad hídrica en el perfil edáfico (Utrilla et al. 2014a) que permitiría un normal crecimiento radicular en los estratos más profundos. En concordancia con lo mencionado y conforme mayor degradación del mallín, los aportes porcentuales de la biomasa radical por profundidad respecto a la biomasa radical total variaron desde los rangos $52-57 \%$ a $0-5 \mathrm{~cm}$ hasta $18-13 \%$ a $10-15 \mathrm{~cm}$ de profundidad bajo las condiciones moderada y muy degradada, respectivamente. En cambio, bajo condición buena la distribución porcentual de la biomasa radical varió entre 26 y $36 \%$ a las mismas profundidades.

\section{Conclusiones}

El trabajo permitió analizar la asociación de variables de la vegetación y el suelo entre sectores y condiciones de degradación de mallines de una cuenca hídrica en la Estepa Magallánica Seca del sur de Santa Cruz. Asimismo, se logró analizar integralmente mediante la prueba factorial bajo estudio los efectos del Sector (S), Fecha (F) y Condición (C), con sus interacciones sobre las variables vegetativas y edáficas. En este contexto, el estudio pudo determinar los efectos principales de $\mathrm{S}$ y de $\mathrm{C}$ sobre las variables de interés y la magnitud de cambio de las mismas entre condiciones del mallín según la interacción CxS. A su vez, el trabajo permitió evaluar la calidad del agua freática y explicar la variación de la biomasa radical entre mallines con diferente condición.

Agradecimientos. El trabajo fue financiado por la Universidad Nacional de la Patagonia Austral (PI 29/A265-1). Agradecemos a los Sres. Tomás O’byrne (Administrador de Ea. Las Horquetas), Mariano Ilaragorri (Propietario de Ea. La Tapera) y al personal conocido de los establecimientos por permitir y colaborar para desarrollar el presente estudio. Además, agradecemos a la Magister Dora Maglione por su colaboración desinteresada en el análisis estadístico de los datos y a los Ing. Boris Diaz, Paola Vargas, Daniela Ferrante y el Dr. Gabriel Oliva por las sugerencias y comentarios para mejorar el presente manuscrito.

\section{Referencias}

Avellaneda, M. O., A. I. Bermejillo, and L. E. Mastrantonio. 2004. Aguas de riego: Calidad y evaluación de su factibilidad de uso. Universidad Nacional de Cuyo, Mendoza. Editorial EDIUNC. Pp. 143.

Ayers, R. S., and D. W. Westcot. 1987. Water quality and use in agriculture. Estudio FAO Riego y Drenaje 29 Rev. 1. FAO. Rome. Italy.

Belsky, A. J., A. Matzke, and S. Uselman. 1999. Survey of livestock influences on stream and riparian ecosystems in the West United States. J. of Soil and Water Conservation 54:419-431. URL: https://tinyurl.com/y6qpo2ps.

Bohm, W. 1979. Methods of studying root systems. Ecological Studies 33. Ed. Springer Verlag. Berlin, Germany. Pp. 
188. https://doi.org/10.1007/978-3-642-67282-8.

Bonvisutto, G. L., R. Somlo, J. Ayesa, M. L. Lanciotti, and E. Moricz de Tecso. 1992. La condición de los mallines del área ecológica Sierras y Mesetas de Patagonia. Rev Arg Prod Anim 12(4):391-400.

Bonvisutto, G. L., R. C. Somlo, M. L. Lanciotti, A. González Carteau, and C. A. Busso. 2008. Guías de condición para pastizales naturales de Precordillera, Sierras y Mesetas y Monte Austral de Patagonia. 1a ed. Buenos Aires: INTA EEA Bariloche. Ediciones INTA. Pp. 48. URL: https://tinyurl.com/y36p5148.

Buono, G. 2007. Variación espacial y temporal de la productividad de mallines patagónicos. Tesis de Magister Scientiae. Curso de Postgrado Área de Recursos Naturales. Escuela para Graduados Alberto Soriano. Universidad Nacional de Buenos Aires. Convenio Facultad de Agronomía (UBA) - Instituto Nacional de Tecnología Agropecuaria (INTA). Pp. 58. URL: http://hdl.handle.net/20.500.12123/6896

Caloin, M., B. Clement and S. Herrmann. 1990. Regrowth kinetics of Dactylis glomerata following defoliation. Ann Bot 66:397-405. https://doi.org/10.1093/oxfordjournals.aob.a088041.

Cipriotti, P. A., M. B. Collantes, R. B. Rauber, K. Braun, and C. Escartín. 2018. Inter-annual and seasonal variation of ANNP and leaf nutrient concentration in cold-temperate wetlands of Tierra del Fuego. Wetlands 38:37-49. https: //doi.org/10.1007/s13157-017-0955-8.

Clary, W. P. 1995. Vegetation and soil responses to grazing simulation on riparian meadows. J Range Manage 48:18-25. URL: https://tinyurl.com/yy3xbobm. https://doi.org/10.2307/4002499.

Collantes, M., and A. M. Faggi. 1999. Los humedales del sur de Sudamérica. En: A.I. Malvárez (ed.). Tópicos sobre humedales subtropicales y templados en Sudamérica. UNESCO. Montevideo Pp. 15-25. URL: http:// www.unesco.org.uy/mab/documentospdf/2.pdf.

Collantes, M., C. Escartín, K. Braun, A. Cingolani, and J. Anchorena. 2013. Grazing and grazing exclusion along a resource gradient in Magellanic meadows of Tierra del Fuego. Rangeland Ecol and Manage 66(6):688-699. URL: https://doi.org/10.2111/REM-D-11-00201.1.

Cremona, M. V., M. L. Lanciotti, and C. L. Bonvissuto. 1996. Dinámica del agua en mallines con diferente condición de pastizal en Patagonia Norte. Actas del XV Congreso Argentino del Suelo. Santa Rosa, La Pampa. 19 al 24 de mayo.

Curll, M., and R. Wilkins. 1983. The comparative effects of defoliation, treading and excreta on a Lolium perenneTrifolium repens pasture grazed by sheep. The Journal of Agricultural Science 100(2):451-460. https://doi.org/10.1017/ S002185960003361X.

Del Valle, H. F. 1993. Mallines de ambiente árido. Pradera salina y estepa arbustivo-graminosa en el NO del Chubut. Pp. 31-39 en J. M. Paruelo, M. B. Bertiller, T. M. Schichter and F. R. Coronato (eds.). Secuencias de deterioro en distintos ambientes patagónicos. Su caracterización mediante el modelo de estados y transiciones. INTA-GTZ.

Dewis, J., and F. Freitas. 1984. Métodos físicos y químicos de análisis de suelos y aguas. Boletín de Suelos de la FAO 10, Organización de las Naciones Unidas para la Agricultura y la Alimentación. Roma. Pp. 252.

Díaz, B. G., O. Bonfili, M. C. Monserrat, L. Almonacid, and S. Sturzenbaum. 2016. Red de Cooperación Hidrometeorológica en Santa Cruz. RALDA 2016. XVI Reunión Argentina y VIII Latinoamericana de Agro-meteorología. Puerto Madryn. Chubut (Argentina). Actas AT4-021: 78-79.

Enriquez, A. S., R. A. Chimner, M. V. Cremona, P. Dielhl, and G. L. Bonvissuto. 2015. Grazing intensity levels influence $\mathrm{C}$ reservoirs of wet and mesic meadows along a precipitation gradient in Northern Patagonia. Wetland Ecol Manage 23:439-451. http://dx.doi.org/10.1007/s11273-014-9393-z.

Enriquez, A. S., and M. V. Cremona. 2018. Particulate organic carbon is a sensitive indicator of soil degradation related to overgrazing in Patagonian wet and mesic meadows. Wetland Ecol Manage 26:345-357. https://doi.org/10.1007/ s11273-017-9577-4.

Flores, E. 1999. La planta, estructura y función. Cartago, C.R. LUR. Pp. 884.

Gabriels, D., and D. Lobo L. 2022. Métodos para determinar granulometría y densidad aparente del suelo. Venesuelos 14:37-48. URL: https://tinyurl.com/y5sybnjh.

Heanet, D. L. 1984. Determination of total organic C in soils by an improved chromic acid digestion and spectrophotometric procedure. Communications in Soil Science and Plant Analysis 15:1191-1213. https://doi.org/ $10.1080 / 00103628409367551$.

Horne, F. 2009. Manejo hidrológico de mallines: manejo sustentable del Mallín Genoa. 1a ed. Neuquén: EDUCO Universidad Nacional del Comahue. Pp. 62.

Jensen, W. 1994. Botánica. Segunda Edición. Distrito Federal, MX. McGraw-Hill. Pp. 762.

Jiménez Rodríguez, C., and D. Arias Aguilar. 2004. Distribución de la biomasa y densidad de raíces finas en un gradiente sucesional de bosques en la Zona Norte de Costa Rica. Revista Forestal Mesoamericana Kurú 1(2):44-63.

Kauffman, J. B., and W. C. Krueger. 1984. Impacts on Riparian Ecosystems and Streamside Management Implications... A Review. J Range Manage 37(5):430-437. URL: https://tinyurl.com/yxjmz9hj. https://doi.org/10.2307/3899631.

Levy, E., and D. Madden. 1933. The point method of pasture analysis. New Zealand Journal of Agricultural 46:267269.

López Bautista, E. A., and B. H. González Ramírez. 2013. Diseño y Análisis de Experimentos. Fundamentos y Aplicaciones en Agronomía. $2^{\circ}$ Edición. Universidad de San Carlos de Guatemala. Facultad de Agronomía. Guatemala.

Mazzoni, E., and M. Vázquez. 2004. Ecosistemas de mallines y paisajes de la Patagonia Austral (Provincia de Santa Cruz). Ediciones INTA. Pp. 63.

Ormaechea, S., V. Utrilla, D. Suárez, and P. L. Peri. 2010. Evaluación objetiva de la condición de mallines de Santa Cruz. Carpeta Técnica EEA INTA Santa Cruz, Sección 9. Producción Animal. Edición EEA Santa Cruz. Pp. 47-52. 
Orloci, L. 1978. Multivariate analysis in vegetation research. Junk Publications, The Hague. Pp. 451.

Peri, P. L., and L. Monelos. 1997. Forestación con estacones de salicáceas en distintos mallines de la provincia de Santa Cruz, Argentina. Actas II Congreso Forestal Argentino y Latinoamericano. Tomo Bosque Cultivado. Pp. 447-456. Posadas, Misiones, 13-15 de Agosto de 1997.

Popolizio, C. A., H. Goetz, and P. L. Chapman. 1994. Short-term response of riparian vegetation to 4 grazing treatments. J Range Manage 47:48-53. URL: https://tinyurl.com/y2tc5awe. https://doi.org/10.2307/4002840.

Radic, S., S. Opazo, E. Mihovilovic, O. Dollenz, and J. Ivelic. 2011. Relación entre la composición botánica con la humedad y densidad aparente del suelo en una vega de Tierra del Fuego. Libro de Resúmenes del XXXVI Congreso de la Sociedad Chilena de Producción Animal. Punta Arenas, Chile. Pp. 139-140.

Raffaele, E. 1999. Mallines: Aspectos Generales y Problemas Particulares. Pp. 25-31 en Malvárez, A. I. (ed.). Tópicos sobre Humedales Templados y Tropicales de Sudamérica. UNESCO, Montevideo, Uruguay. URL: https://tinyurl.com/ yccfvhu8.

Roberson, E. 1996. Impacts of Livestock Grazing on Soils and Recommendations for Management. CNPS Land Management. Pp. 30.

Rodríguez, M. V., M. B. Bertiller, and C. L. Sain. 2006. Spatial patterns and Chemicals characteristics of root biomass in ecosystems of the Patagonian Monte disturbed by grazing. J Arid Environ 70:137-151. https://doi.org/10.1016/ j.jaridenv.2006.12.010.

Sellés, G., R. Ferreyra, R. Ruíz, R. Ferreyra, and R. Ahumada. 2012. Compactación de suelos y su control. Estudio de casos en el Valle de Aconcagua. Boletín INIA No 234. Instituto de Investigaciones Agropecuarias, Centro Regional de Investigación La Platina, Santiago, Chile. Pp. 53.

Standard Methods for the Examination of Water and Wastewater. 21st Edition. 2005. 3500-Pb Lead. American Public Health Association, American Water Works Association, Water Environment Federation. Port City Press, Baltimore, Maryland. Pp: 3-79 a 3-80.

Suárez, D., S. Ormaechea, P. L. Peri, and V. Utrilla. 2010. Caracterización objetiva de la condición en mallines de Santa Cruz. Informe Técnico. INTA EEA Santa Cruz. Pp. 20. URL: https://tinyurl.com/y5kf8xqu.

Thorne, D. W., and H. B. Peterson. 1954. Irrigated Soils. Constable and Company Limited, London. Pp. 113. https: //doi.org/10.1097/00010694-195411000-00021.

Thornton, B., and P. Millard. 1996. Effects of severity of defoliation on root functioning in grasses. J Range Manage 49: 443-447. URL: https://tinyurl.com/y3aar84a. https://doi.org/10.2307/4002927.

Utrilla, V. 2003. Respuesta de la vegetación y de ovejas de refugo en un mallín magallánico bajo diferentes condiciones de uso previo y de asignación de forraje. Tesis de Magíster Scientiae. Curso de Posgrado en Producción Animal. Facultad de Ciencias Agrarias de Balcarce. Universidad Nacional de Mar del Plata. Pp. 91. URL: https://tinyurl.com/ y5fy6em9.

Utrilla, V., M. Brizuela, and A. Cibils. 2005. Riparian habitats (mallines) of Patagonia: a key grazing resource for sustainable sheep farming operations. Outlook on Agriculture 34(1):55-59. https://doi.org/10.5367/0000000053295088.

Utrilla, V. R., M. Andrade, P. L. Peri, and S. L. Billoni. 2012. Evaluación de indicadores de degradación en mallines del Sur de Santa Cruz. Rev Arg Prod Anim 32(1): 376. URL: https://tinyurl.com/yxjzj2dy.

Utrilla, V. R., M. Andrade, P. L. Peri, S. L. Billoni, and B. Rogel. 2013. Evaluación de indicadores físicos de degradación en mallines del Sur de Santa Cruz. En: Actas de las Jornadas Argentinas de Conservación de Suelos 2013. Ciudad Autónoma de Buenos Aires. Copia en CD-Rom. Ediciones INTA. Pp. 2. URL: https://tinyurl.com/y6g712tn.

Utrilla, V. R., M. Andrade, S. Billoni, P. L. Peri, M. V. Sturzenbaum, and B. Rogel. 2014a. Monitoreo de indicadores de degradación en mallines bajo pastoreo ovino en el Sur de Santa Cruz. Informe Técnico. INTA EEA Santa Cruz (Convenio INTA-CAP-UNPA). Pp. 31. URL: https://tinyurl.com/y54m44je.

Utrilla, V., P. L. Peri, S. Billoni, B. Rogel, and M. Andrade. 2014b. Caracterización y evaluación de indicadores de degradación en mallines del Sur de Santa Cruz. En: Bienales del III Encuentro de Investigadores de la Patagonia Austral (ISBN 978-987-3714-00-9). $3^{\circ}$ edición. Unidad Académica Río Turbio de la UNPA. Pp. 27-30.

Vargas, P. P. 2017. Mallines del Sur de la Patagonia. Interacciones entre Unidades Fisiográficas y Productividad en Diversos Ambientes Geomofológicos. Tesis Doctoral en Ciencias Aplicadas. Universidad Nacional de Lujan. Pp. 239. URL: https://tinyurl.com/y37aml8y.

Wilkins, R., and E. A. Garwood. 1986. Effects of Treading, Poaching and Fouling on Grassland Production and Utilization. Session 1. Herbage Production. In: J. Frame (ed.). Grazing. Occasional Symposium № 19. British Grassland Society. Pp. 19-31.

Zerpa, G., O. Sosa, J. Bernardi, J. P. Bolatti, A. Galindo, and J. Maldonado. 2013. Resistencia mecânica a la penetración en pasturas. Agromensajes 35:64-68. URL: https://tinyurl.com/pryncjf. 